\title{
Deployment of a bioabsorbable plate as the rigid buttress for skull base repair after endoscopic pituitary surgery
}

\author{
Qichao Qi ${ }^{1,2,3}$, Yongpeng Zhang ${ }^{1,4}$, Junpeng Wang ${ }^{1}$, Hanlin Zhong ${ }^{1}$, Haijun Chen ${ }^{1}$,Chuanwei Wang ${ }^{1}$, \\ Jiangang Wang ${ }^{1}$, Xiaolan Cai ${ }^{5}$, Lei Sun ${ }^{6}$, Shilei $\mathrm{Ni}^{1,2,3}$
}

${ }^{1}$ Department of Neurosurgery, Qilu Hospital of Shandong University, Jinan, China; ${ }^{2}$ Institute of Brain and Brain-Inspired Science, Shandong University, Jinan, China; ${ }^{3}$ Shandong Key Laboratory of Brain Function Remodeling, Jinan, China; ${ }^{4}$ Department of Neurosurgery, Rizhao Central Hospital, Rizhao, China; ${ }^{5}$ Department of Otorhinolaryngology, Qilu Hospital of Shandong University, Jinan, China; ${ }^{6}$ Department of Endocrinology, Qilu Hospital of Shandong University, Jinan, China

Contributions: (I) Conception and design: Q Qi, S Ni; (II) Administrative support: All authors; (III) Provision of study materials or patients: All authors; (IV) Collection and assembly of data: Q Qi, Y Zhang, L Sun, S Ni; (V) Data analysis and interpretation: Q Qi, L Sun, S Ni; (VI) Manuscript writing: All authors; (VII) Final approval of manuscript: All authors.

Correspondence to: Shilei Ni, MD, PhD. Department of Neurosurgery, Qilu Hospital of Shandong University, Wenhuaxi Road 107, Jinan 250012, Shandong, China. Email: nishilei@sdu.edu.cn; Lei Sun, MD, PhD. Department of Endocrinology, Qilu Hospital of Shandong University, Wenhuaxi Road 107, Jinan 250012, China. Email: 13969193236@163.com.

Background Bioresorbable alloplastic implants have become desirable as a rigid buttress for reconstructing skull base defects. This study aimed to describe the use of a biodegradable plate (PolyMax RAPID) in skull base repair of endoscopic endonasal pituitary surgery and to investigate the clinical outcome and safety of this novel method.

Methods: Between January 2019 and January 2020, 22 patients with pituitary adenomas who underwent endoscopic skull base repair with a Polymax RAPID plate were included. After endonasal transsphenoidal surgery, a trimmed bioresorbable plate was placed in the position between the dura and the bone of the skull base to reconstruct the sellar floor and buttress the pituitary gland and sellar packing. The patient demographics, radiologic imaging, and postoperative outcomes were carefully reviewed. All patients were followed up by a routine nasal endoscopic assessment and radiologic examinations.

Results: The present study comprised 10 (45.5\%) males and $12(54.4 \%)$ females with an average age of 51.9 years. There were 7 (31.8\%) growth hormone (GH) secreting adenomas, 2 (9.1\%) thyroid stimulating hormone (TSH) secreting adenomas, and 13 (59.1\%) non-functioning adenomas. Enlarged sellar floor and paranasal sinusitis were seen in $13(59.1 \%)$ and $11(50.0 \%)$ cases shown by preoperative computed tomography (CT) or magnetic resonance imaging (MRI), respectively. There were $6(27.3 \%)$ grade-1 and $16(72.7 \%)$ grade- 0 cases by intraoperative cerebrospinal fluid (CSF) leak grading. None of these patients received lumbar drains postoperatively and no postoperative CSF rhinorrhea was detected in our series. The PolyMax RAPID plates which could be clearly identified on postoperative CT or sagittal T1-weighted MRI were shown to provide an ideal rigid buttress for sellar repair.

Conclusions: The Polymax RAPID plate can be an optimal implant to achieve rigid repair of sellar floor defects after endonasal transsphenoidal pituitary surgery.

Keywords: Endoscopic transsphenoidal surgery; skull base reconstruction; bioabsorbable material; pituitary adenomas

Submitted Jul 31, 2020. Accepted for publication Jan 11, 2021.

doi: $10.21037 / g s-20-642$

View this article at: http://dx.doi.org/10.21037/gs-20-642 


\section{Introduction}

Over the last 2 decades, the endoscopic transsphenoidal approach has been widely accepted as the predominant method for resection of pituitary adenomas and sellar lesions around the world $(1,2)$. A reliable skull base reconstruction after endoscopic pituitary surgery is necessary since insufficient repair can be associated with cerebrospinal fluid (CSF) leakage, meningitis, and pneumocephalus $(3,4)$. Numerous reconstruction methods have been introduced with a variety of materials, each with particular advantages and disadvantages regarding efficacy, postoperative complications, and strength $(5,6)$.

The addition of a rigid buttress to skull base reconstruction should be considered because it provides structural support in holding repair materials in position and prevents graft migration by intracranial pulsation and gravity (5-7). PolyMax RAPID (Synthes; Oberdorf, Switzerland), which is manufactured from $85: 15$ poly L-lactide-co-glycolide, has been approved for craniofacial reconstruction. There have been multiple studies for its use in zygomaticomaxillary complex and orbital floor fracture repair $(7,8)$. It can maintain $85 \%$ of its initial bending strength at 8 weeks. Within approximately 12 months of placement, the material gradually loses integrity and breaks down into small particles. The polymer is broken down into lactic and glycolic acids, which are eventually eliminated through natural body metabolism without toxic tissue accumulation $(7,9)$.

The method of skull base closure using this material after endoscopic pituitary surgery was first described in 2013 (7). However, in that report, there were no endoscopic examinations or radiological imaging and only 4 patients were included. The objective of this study was to evaluate the outcome of skull base reconstruction using this novel bioabsorbable plate in patients undergoing endoscopic pituitary surgery. The technique of sellar repair using a PolyMax RAPID plate as a rigid buttress is described. We present the following article in accordance with the STROBE reporting checklist (available at http://dx.doi. org/10.21037/gs-20-642).

\section{Methods}

\section{Patient population}

We performed a retrospective review on all patients who underwent endoscopic pituitary surgery with skull base repair with a Polymax RAPID plate at the Department of
Neurosurgery at Qilu Hospital of Shandong University between January 2019 and January 2020. This study was approved by the institutional ethics committee of Qilu Hospital of Shandong University (KYLL-2017(KS)-090), and was performed in accordance with the principles of the Declaration of Helsinki (as revised in 2013). The requirement of obtaining individual consent was waived for this retrospective analysis.

\section{Perioperative evaluation and intraoperative CSF leakage grading}

Tumor size was determined by preoperative magnetic resonance imaging (MRI). Computed tomography (CT) of paranasal sinuses was also done for all cases preoperatively. Medical records were reviewed for patient demographics, tumor characteristics, perioperative variables, incidence of complications, and postoperative outcomes. Intraoperative CSF leaks were categorized into grades $0,1,2$, or 3 based on Kelly's classification $(6,10)$. The clinical data of these patients were separately reviewed by 2 neurosurgeons (QQ and $\mathrm{YZ}$ ). Inconsistent results were confirmed and recorded together with the review of a third neurosurgeon (SN).

\section{Surgical procedure and repair protocol}

All pituitary adenoma patients underwent tumor removal through an endoscopic endonasal transsphenoidal approach. Bilateral sphenoidotomy was performed and the posterior of the nasal septum, rostrum of the sphenoid bone, and any obstructive septations in the sphenoid sinus were resected. After tumor resection, sellar defect closure was performed by a multilayer repair (Figure 1). An artificial dural substitute (DuraMax, TianXinFu; Beijing, China) was positioned first in the epidural space. The gelatin sponges were then wedged into the sellar space as a plug. The above procedures are the standard reconstruction method used prior to introducing this bioresorbable plate. After this, a PolyMax plate was deployed in the space with two ends between the dura and bone edges of the sellar defect. The plate was $1.2 \mathrm{~mm}$ thick and can be easily bent and trimmed with scissors after submerging in a $70{ }^{\circ} \mathrm{C}$ water bath. The defect size was estimated using a cottonoid in situ and the plate was subsequently shaped into a slight convexity with the fit size. It is transparent with some flexibility retained, which preserves visualization of the defect margins and underlying tissue during positioning and decreases the potential risk of injury to neurovascular structures. The 

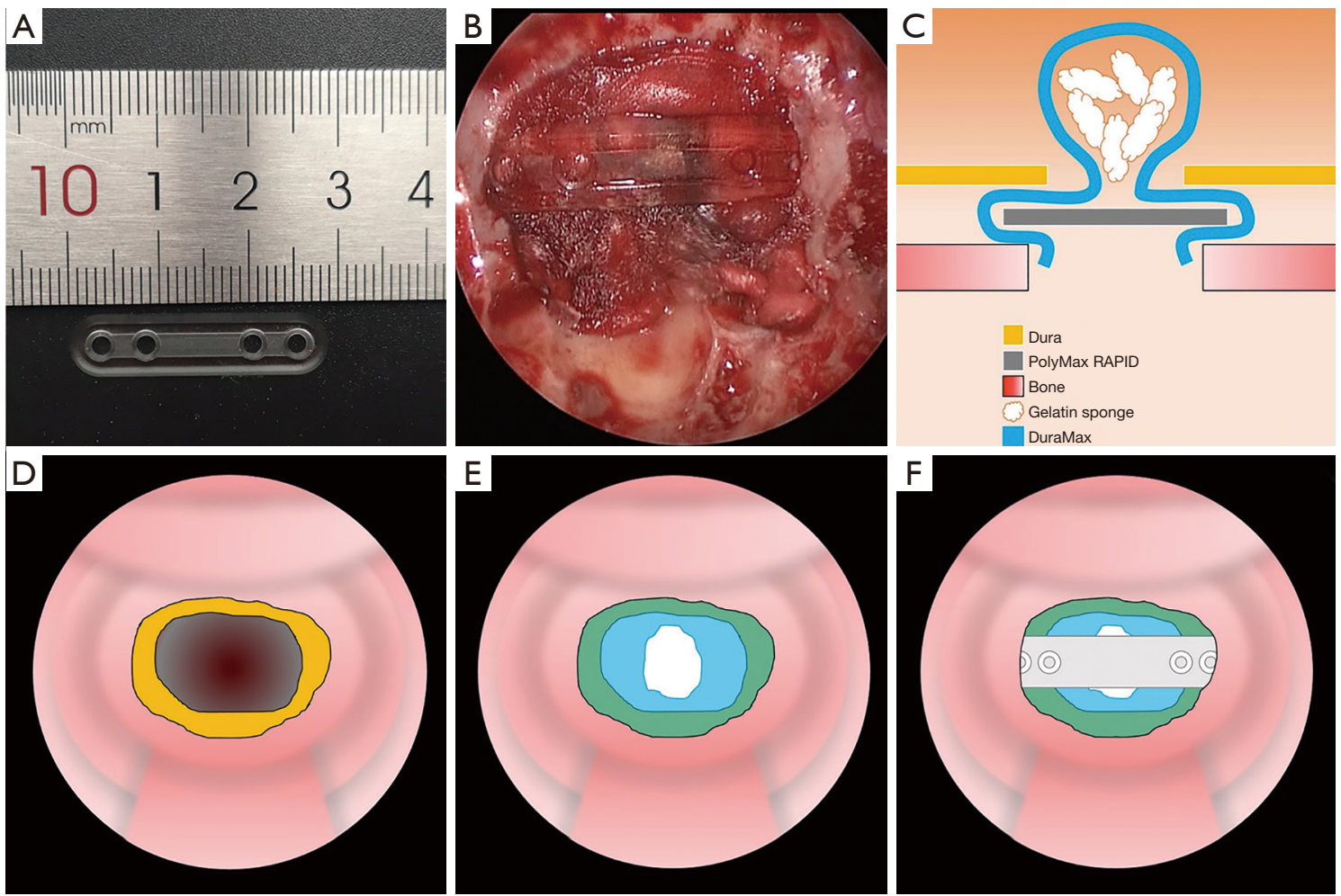

Figure 1 The PolyMax plate and its deployment in sellar repair. (A) Photograph of the bioresorbable plate used in this series. (B) Intraoperative image showing the deployment of the PolyMax plate. (C) Schematic drawing of the coronal view of the present sellar repair technique. (D,E,F) Schematics illustrating the reconstruction procedure in this study (yellow, dura; white, gelatin sponge; red, sellar bone; blue, DuraMax; and grey, PolyMax plate).

free mucosal flap dissected from the sphenoid sinus was used to cover the sellar floor. An on-lay cover with the SURGICEL fibrillar absorbable hemostat (Ethicon; San Lorenzo, Puerto Rico, USA) and gelatin sponge were subsequently used. Iodoform gauzes and Suntouch sponges (Foryou Medical Devices; Huizhou, China) were placed in the sphenoid sinus and nasal cavity for 3 days as a temporary buttress. Postoperative CT at the first day after surgery was conventionally performed to exclude intrasellar hemorrhaging and to identify the position of the PolyMax plate. In all cases, prophylactic intravenous antibiotics were administered 30 minutes preoperatively for 48 hours following surgery as standard of practice at our institution.

\section{Follow-up}

The postoperative nasal endoscopic assessment and debridement if needed were performed by the otolaryngologist at 1 month after surgery. The patients were also followed up by appointed clinicians to do routine radiological examinations at 3, 6, and 12 months after surgery. Postoperative adverse events, such as CSF rhinorrhea, infection, hemorrhage, headache, or nasal discomfort, were recorded if present.

\section{Results}

Twenty-two patients who underwent skull base repair with the PolyMax RAPID bioabsorbable plate were identified by the retrospective review. All cases were pituitary adenomas resected by purely endoscopic endonasal transsphenoidal approaches. The clinical characteristics of patients included are listed in Table 1. The present study comprised 10 (45.5\%) males and 12 (54.4\%) females. The mean age of the patients was 51.9 years ranging from 18 to 68 years. The mean body mass index (BMI) of the patients was 24.6 ranging from 20.4 to 30.4 . This series consisted of $7(31.8 \%)$ growth hormone $(\mathrm{GH})$ secreting adenomas, $2(9.1 \%)$ thyroid 


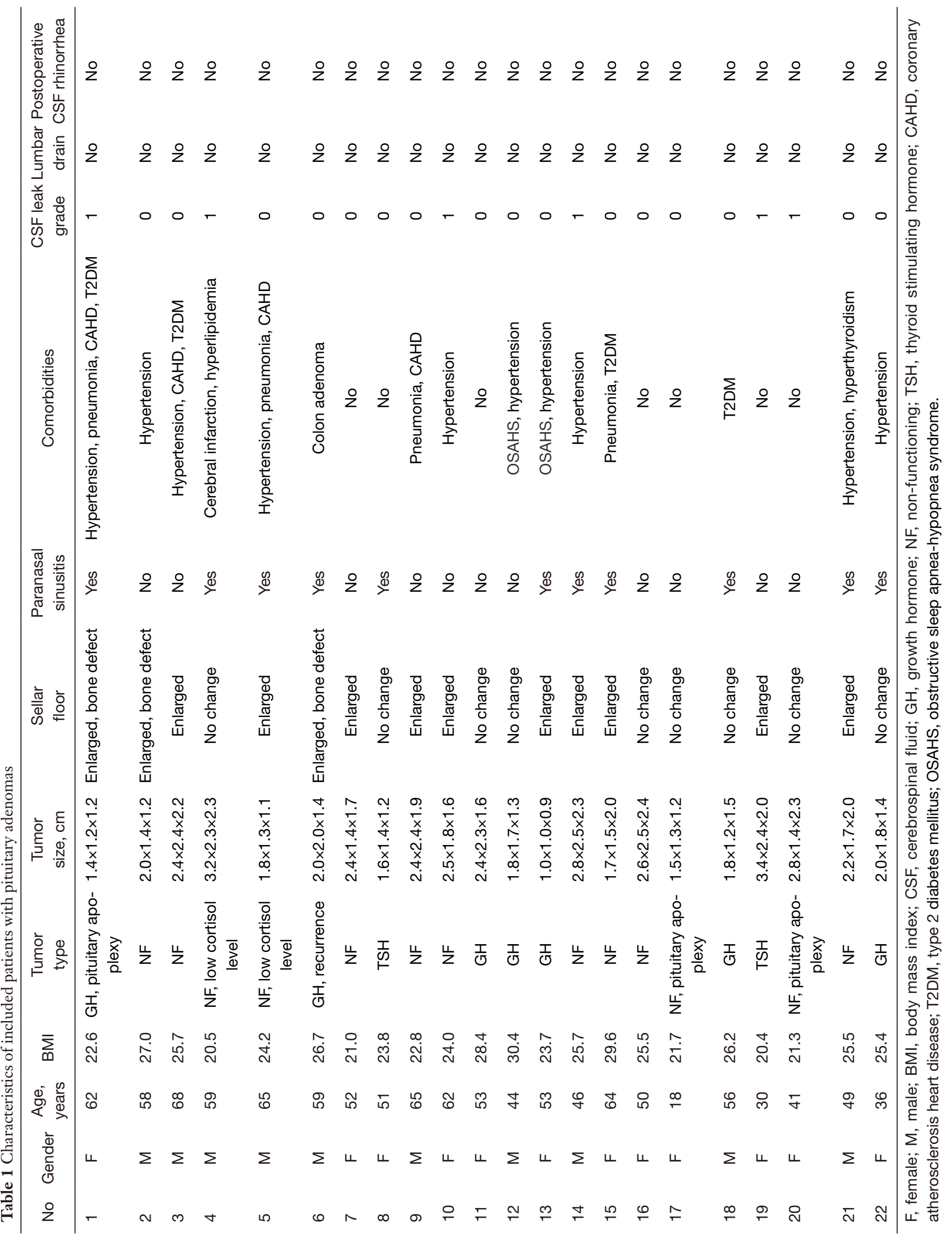



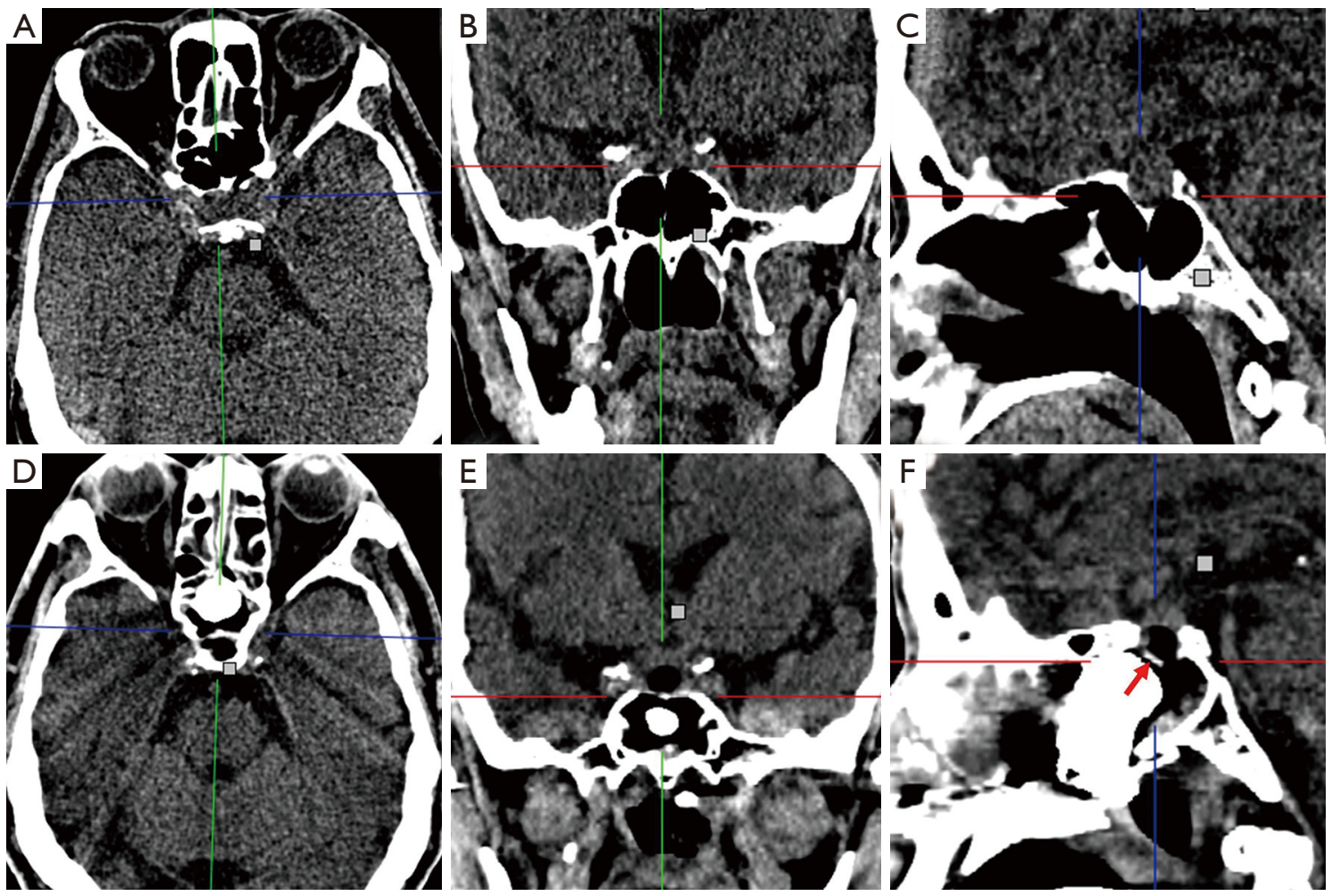

Figure 2 Preoperative and postoperative computed tomography (CT) scans identifying the position of a PolyMax plate. The red arrow indicates the PolyMax plate. (A) Axial, (B) coronal, and (C) sagittal views of preoperative CT images. (D) Axial, (E) coronal, and (F) sagittal views of postoperative CT images showing the position of the PolyMax plate (soft tissue window with 70-90 Hounsfield units).

stimulating hormone (TSH) secreting adenomas, and 13 (59.1\%) non-functioning adenomas. Among these patients, there was $1(4.5 \%)$ recurrent pituitary adenoma and 3 $(13.6 \%)$ cases with pituitary apoplexy. An enlarged sellar floor and paranasal sinusitis were seen in $13(59.1 \%)$ and $11(50.0 \%)$ cases by preoperative CT or MRI, respectively. Comorbidities included hypertension, pneumonia, coronary atherosclerotic heart disease, type 2 diabetes mellitus (T2DM), cerebral infarction, hyperlipidemia, colon adenoma, obstructive sleep apnea-hypopnea syndrome (OSAHS), and hyperthyroidism. There were 6 (27.3\%) grade- 1 and $16(72.7 \%)$ grade- 0 cases by intraoperative CSF leak grading. None of these patients received lumbar drains postoperatively and no postoperative CSF rhinorrhea was detected in our series.

The position of the PolyMax RAPID plate could be clearly identified on CT or sagittal T1-weighted MRI images (Figure 2). The postoperative radiologic examinations showed no horizontal or vertical migration of the implants (Figure 3). All patients underwent nasal endoscopic assessment and debridement 1 month after surgery. None of the included patients were noted to have epistaxis, CSF rhinorrhea, or local inflammatory reactions. Complaints of slight nasal stuffiness and tolerable headache were observed in 3 (13.6\%) and 2 (9.1\%) cases respectively during follow-up.

\section{Discussion}

To the best of our knowledge, the bioresorbable miniplate manufactured from 85:15 poly L-lactide-co-glycolide was first introduced as a rigid buttress for sellar repair of 4 patients by Tabaee et al. (7). That is also the only publication regarding the deployment of this bioabsorbable implant after endoscopic pituitary surgery. Our study provides the largest number of cases to date and makes an important contribution to the field by summarizing their clinical characteristics, radiologic features, and the efficacy and safety of skull base reconstruction using PolyMax RAPID. 

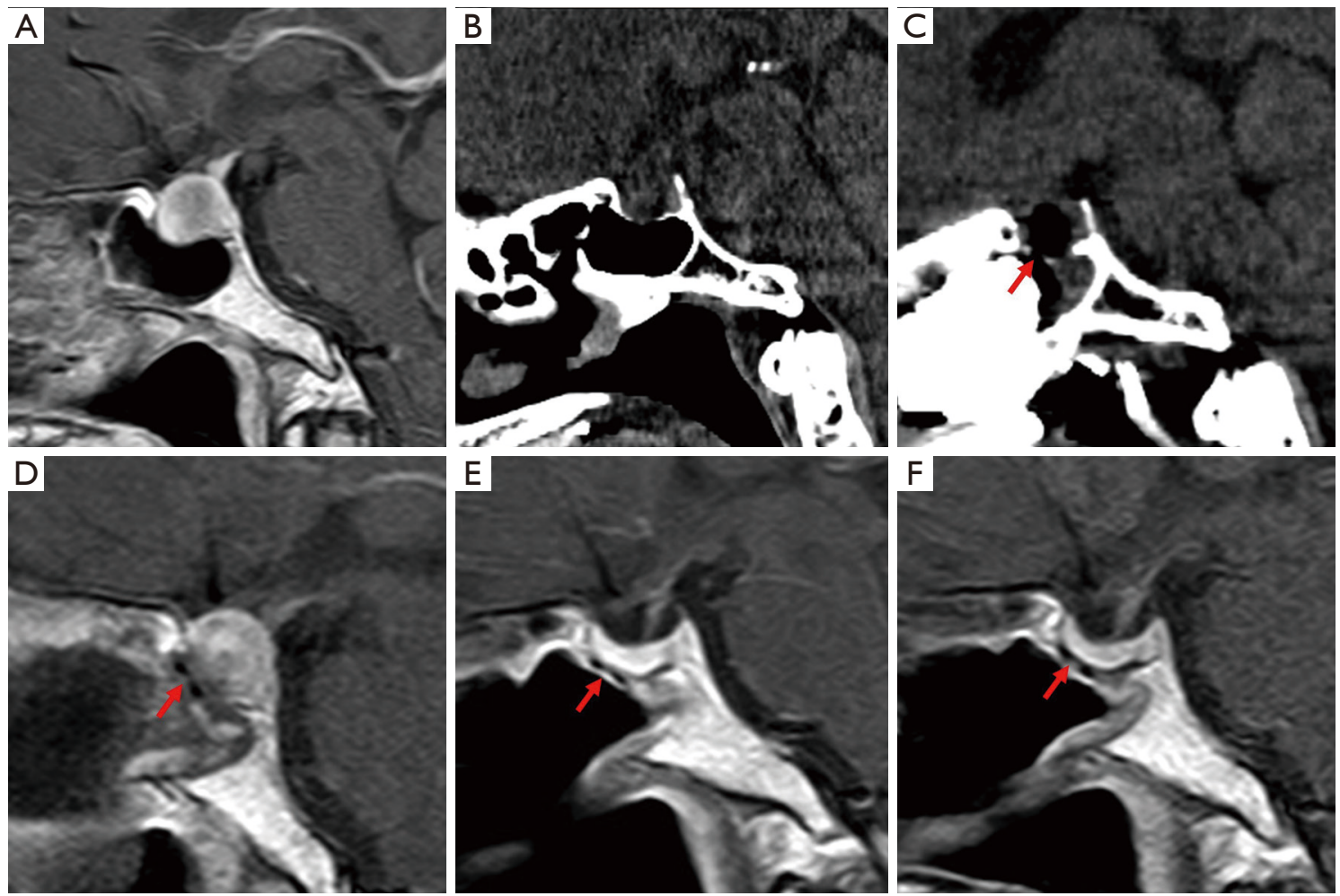

Figure 3 Radiologic images showing no migration of the implants during follow-up. The red arrow indicates the PolyMax plate. (A) T1 contrast-enhanced sagittal view of preoperative magnetic resonance imaging (MRI). (B) Sagittal view of preoperative computed tomography (CT) image. (C) Sagittal view of CT scan at the 1st day after surgery. (D) T1 contrast-enhanced sagittal view of MRI at the 5th day after surgery. T1 contrast-enhanced sagittal view of MRI performed 3 (E) and 6 (F) months after surgery showing no migration of the implant.

Skull base repair after endoscopic pituitary surgery, which mainly consists of intrasellar packing and reconstruction of the sellar floor, is vital for the patient's quality of life. If the reconstruction of the sellar floor is inadequate, intrasellar packing may migrate by CSF pulsation and gravity, leading to an increased risk of CSF rhinorrhea or meningitis. We prefer to apply a rigid material to reconstruct the sellar floor because it can efficiently support packing materials in the sellar cavity, especially in cases with a bone defect of the sellar floor or an empty sellar preoperatively; a comorbidity including hypertension, pneumonia, or T2DM; or high BMI.

The use of autologous bone fragments from the middle turbinate, nasal septum, and anterior sphenoid wall presents some problems such as irregular size, thickness, and border (11). Alloplastic rigid materials such as titanium, porous polyethylene (MEDPOR), and silicone are characterized by strong tensile strength and good stability and are readily available and easy to handle (3,12-15). However, permanent foreign buttress which undergo minimal or no resorption are difficult to remove at reoperation and relatively susceptible to infection, hemorrhage, migration, and exposure over time. A potential risk of injury to neurovascular structures by titanium during positioning should also be noted (6). Silicone plates were reported to induce a local inflammatory reaction postoperatively (16). Therefore, bioresorbable alloplastic implants, which offer a useful alternative in the reconstruction of skull base defects, have been increasingly popular. Currently available bioresorbable implants, including Resorb-X, MacroSorb, polydioxanone flexible plates, and LactoSorb, have been reported for endoscopic skull base repair $(5,11,17,18)$.

Compared to these studies, the PolyMax plate has the following advantages. It has (I) adequate tensile strength for a rigid buttress, is (II) easily bent by a heated water bath and can be trimmed with scissors to fit the defect, (III) retains some flexibility which decreases the potential risk of injury to neurovascular structures during positioning, (IV) is easily identified by $\mathrm{CT}$ or MRI with no radiologic artifact, $(\mathrm{V})$ can 
be easily removed at reoperation through the nostrils, and (VI) is bioresorbable material with good biocompatibility.

A few limitations in this study should also be addressed. The cases in our series are mainly grades 0 and 1 CSF leaks. Although we had a $100 \%$ success rate in our skull base repairs, the efficacy of this method for high-flow CSF leakage remains unclear. Our series was additionally limited to sellar repair of endoscopic pituitary surgery and therefore it does not investigate the role of sellar repair using this biodegradable plate for other types of skull base defects or parasellar lesions.

\section{Conclusions}

The findings of this retrospective study imply that the PolyMax plate provides an alternative option for skull base reconstruction after endoscopic endonasal pituitary surgery. It may be considered for a variety of sellar or anterior cranial fossa defects. The long-term outcomes and complication rates need to be evaluated with further data.

\section{Acknowledgments}

We would like to thank Editage (www.editage.cn) for English language editing.

Funding: This study was provided by the National Natural Science Foundation of China (grant No. 81874082 and No. 81472353), Key Research and Development Plan of Shandong Province (grant No. 2016GSF201014 and No. 2018GSF118094), Shandong Provincial Natural Science Foundation (grant No. ZR2020QH234), and Jinan Science and Technology Development Plan (grant No. 201821049).

\section{Footnote}

Reporting Checklist: The authors have completed the STROBE reporting checklist. Available at http://dx.doi. org/10.21037/gs-20-642

Data Sharing Statement: Available at http://dx.doi. org/10.21037/gs-20-642

Peer Review File: Available at http://dx.doi.org/10.21037/gs20-642

Conflicts of Interest: All authors have completed the ICMJE uniform disclosure form (available at http://dx.doi. org/10.21037/gs-20-642). The authors have no conflicts of interest to declare.

Ethical Statement: The authors are accountable for all aspects of the work in ensuring that questions related to the accuracy or integrity of any part of the work are appropriately investigated and resolved. This study was approved by the institutional ethics committee of Qilu Hospital of Shandong University [KYLL-2017(KS)-090], and was performed in accordance with the principles of the Declaration of Helsinki (as revised in 2013). The requirement of obtaining individual consent was waived for this retrospective analysis. The authors declare that the patients' personal data have been secured.

Open Access Statement: This is an Open Access article distributed in accordance with the Creative Commons Attribution-NonCommercial-NoDerivs 4.0 International License (CC BY-NC-ND 4.0), which permits the noncommercial replication and distribution of the article with the strict proviso that no changes or edits are made and the original work is properly cited (including links to both the formal publication through the relevant DOI and the license). See: https://creativecommons.org/licenses/by-nc-nd/4.0/.

\section{References}

1. de Divitiis E, Laws ER, Giani U, et al. The current status of endoscopy in transsphenoidal surgery: an international survey. World Neurosurg 2015;83:447-54.

2. Zhang M, Singh H, Almodovar-Mercado GJ, et al. Required Reading: The Most Impactful Articles in Endoscopic Endonasal Skull Base Surgery. World Neurosurg 2016;92:499-512.e2.

3. Garcia-Navarro V, Anand VK, Schwartz TH. Gasket seal closure for extended endonasal endoscopic skull base surgery: efficacy in a large case series. World Neurosurg 2013;80:563-8.

4. Lobatto DJ, de Vries F, Zamanipoor Najafabadi AH, et al. Preoperative risk factors for postoperative complications in endoscopic pituitary surgery: a systematic review. Pituitary 2018;21:84-97.

5. Potter NJ, Graham SM, Chang EH, et al. Bioabsorbable plate cranial base reconstruction. Laryngoscope 2015;125:1313-5.

6. Esposito F, Dusick JR, Fatemi N, et al. Graded repair of cranial base defects and cerebrospinal fluid leaks in transsphenoidal surgery. Oper Neurosurg (Hagerstown) 2007;60:295-303; discussion 303-4. 
7. Tabaee A, Kamat A, Shrivastava R. Complex reconstruction of the sella using absorbable mini-plate in revision endoscopic pituitary surgery: technical note. J Neurol Surg A Cent Eur Neurosurg 2013;74:313-7.

8. Chu SG, Lee JS, Lee JW, et al. Comparisons among four types of absorbable plates used for internal fixation of zygomaticomaxillary complex fractures. J Craniomaxillofac Surg 2019;47:383-8.

9. Young SM, Sundar G, Lim TC, et al. Use of bioresorbable implants for orbital fracture reconstruction. Br J Ophthalmol 2017;101:1080-5.

10. Conger A, Zhao F, Wang X, et al. Evolution of the graded repair of CSF leaks and skull base defects in endonasal endoscopic tumor surgery: trends in repair failure and meningitis rates in 509 patients. J Neurosurg 2018;130:861-75.

11. Cavallo LM, Messina A, Esposito F, et al. Skull base reconstruction in the extended endoscopic transsphenoidal approach for suprasellar lesions. J Neurosurg 2007;107:713-20.

12. Cappabianca P, Cavallo LM, Mariniello G, et al. Easy sellar reconstruction in endoscopic endonasal transsphenoidal surgery with polyester-silicone dural

Cite this article as: Qi Q, Zhang Y, Wang J, Zhong H, Chen H, Wang C, Wang J, Cai X, Sun L, Ni S. Deployment of a bioabsorbable plate as the rigid buttress for skull base repair after endoscopic pituitary surgery. Gland Surg 2021;10(3):10101017. doi: $10.21037 /$ gs-20-642 substitute and fibrin glue: technical note. Neurosurgery 2001;49:473-5; discussion 475-6.

13. Park J, Guthikonda M. The Medpor sheet as a sellar buttress after endonasal transsphenoidal surgery: technical note. Surg Neurol 2004;61:488-92; discussion 493.

14. Biron VL, Gross M, Broad R, et al. Radial forearm free flap with titanium mesh sandwich reconstruction in complex anterior skull base defects. J Craniofac Surg 2012;23:1763-5.

15. Leng LZ, Brown S, Anand VK, et al. "Gasket-seal" watertight closure in minimal-access endoscopic cranial base surgery. Neurosurgery 2008;62:ONSE342-ONSE3.

16. Matsumoto K, Kohmura E, Tsuruzono K, et al. Silicone plate-induced granuloma presenting pituitary apoplexylike symptoms: case report. Surg Neurol 1995;43:166-9.

17. Kaptain GJ, Vincent DA, Laws ER, Jr. Cranial base reconstruction after transsphenoidal surgery with bioabsorbable implants. Neurosurgery 2001;48:232-3; discussion 233-4.

18. Al-Asousi F, Okpaleke C, Dadgostar A, et al. The use of polydioxanone plates for endoscopic skull base repair. Am J Rhinol Allergy 2017;31:122-6. 